\title{
La transformación biopólítica del Estado-Nación "El arte de gobernar a las poblaciones en el
} neoliberalismo"

The bio-political transformation of the Nation-State "The art of governing populations in neoliberalism"

Gustavo García Rojas ${ }^{1}$

DOI: $\underline{\text { http://dx.doi.org/10.29105/pgc4.7-2 }}$

Universidad Autónoma de Nuevo León

(D) https://orcid.org/0000-0001-6502-0684

\section{RESUMEN}

El presente artículo es producto de una revisión bibliográfica, cuyo objetivo consistió en debatir sobre las transformaciones conceptuales clave en lo referente al papel del estado o el "arte de gobernar" desde la postura crítica de la biopolítica. Se aplicó el método de análisis, con un enfoque cualitativo, diseño no experimental, bajo un nivel documental-bibliográfico transversal. La revisión documental construye una articulación entre este cambio de concepciones histórico con las formulaciones críticas de la colonialidad y la forma política del estado de excepción en la trayectoria de transformaciones que ha tenido el papel del estado-nación como forma privilegiada de poder en el capitalismo. En el presente capitulo se abordarán estas cuestiones de manera general. Se concluye con la introducción de la concepción de Necrocapitalismo para comprender la forma en que los estados controlan a sus poblaciones como una determinación del cálculo económico global en el mundo contemporáneo.

Palabras clave: Biopolitica, Colonialidad, Estado de Excepción, Necrocapitalismo.

\section{ABSTRACT}

This article is the product of a bibliographic review, which objective was to discuss the key conceptual transformations regarding the role of the state or the "art of governing" from the critical stance of biopolitics. The method of analysis was applied, with a qualitative approach, non-experimental design, under a transversal documentary-bibliographic level. After the documentary review it was found that: this change of historical conceptions is articulated with the critical formulations of coloniality and the political form of the state of exception in the path of transformations that the role of the nation-state as a privileged form of power in the capitalism has had. This will be addressed in a general way in this chapter. It is concluded that the conception of Necrocapitalism is introduced to understand the way in which states control their populations as a determination of the global economic calculation in the contemporary world.

Key words: Biopolitics, coloniality, state of exception, necrocapitalism.

Cómo referenciar este artículo:

García Rojas, G. (2018). a transformación biopólítica del Estado-Nación "El arte de gobernar a las poblaciones en el neoliberalismo". Revista Política, Globalidad y Ciudadanía, 31-41. Recuperado de http://revpoliticas.uanl.mx/ index.php/RPGyC/article/view/87

Recibido: 16 de Julio 2017 - Aceptado: 08 de Septiembre 2017

\section{(cc) BY-NC-ND}

1 Doctor en Ciencias Sociales, con especialización en Relaciones de Poder y Cultura Política, en la UAM-Xochimilco

Revista Política, Globalidad y Ciudadanía, Vol. 4 No. 7, Enero - Junio 2018, Universidad Autónoma de Nuevo León, Monterrey, México, ISSN 2395-8448. pp 31-41. http://revpoliticas.uanl.mx/index.php/RPGyC/article/view/87 


\section{1.- INTRODUCCIÓN}

Controlar y regular la gestión de la población es uno de los efectos del proceso que Michel Foucault describe como la transición de una sociedad de soberanía a una sociedad de control y la transformación de la gestión de la tarea de gobernar que trae aparejado éste. Ubicado en el siglo XVI en la Europa occidental, dicho proceso consistió en lo que el filósofo francés caracterizó como una interiorización de las tareas de gobierno, a diferencia de las formas de las sociedades de soberanía anteriores al siglo XVI (y a la construcción de estados monárquicos centralizados) que concebían a las tareas de gobernar como un acto exterior ${ }^{2}$ y al estado como fuente exclusiva o predominante de poder. Para Foucault, la tarea de gobierno puede ubicarse en una pluralidad de espacios (la familia, el trabajo, el estado) por lo que el poder puede entonces ubicarse en una gama amplia de ámbitos y no puede ubicársele en un solo lugar privilegiado - e.g. el estado 3 .

Tanto o más importante que lo anterior, es un efecto que contiene una importancia toral: el predominio del modelo de la economía (racionalidad, cuantificación, estadística) en la gestión de gobierno, se instituye en esta época también, coincidente con el surgimiento de la economía política, predominio que ha permanecido en forma intermitente a lo largo de dos siglos.

La discusión entonces, sobre lo que Foucault llama "gubernamentalidad" como paradigma del "arte de gobernar" es decir, de controlar y gestionar las poblaciones y las distintas esferas de la vida, se ubica en el ámbito político contemporáneo en el inicio de diversas teorizaciones académicas (y prácticas de gobierno) de la economía que él llama indistintamente "liberales" y "neoliberales" en Alemania y Estado Unidos, al final de la segunda guerra mundial ${ }^{4}$.

Estas teorizaciones surgen como una reacción al predominio de políticas económicas intervencionistas y estatistas en buena parte del mundo de posguerra, identificadas con la figura de John M. Keynes. Foucault analiza al diseño del estado alemán pos-nazismo y de la teorización económica de la escuela de Chicago en EE. UU como modelos de "fobia al estado" ${ }^{5}$ en los que se teoriza al estado interventor como mutilador de libertades esenciales y obstructor del desempeño adecuado de la economía.

En el caso norteamericano específicamente, representado por los economistas de Chicago, el modelo busca "extender la racionalidad del mercado, los esquemas de análisis que éste propone y los criterios de decisión que sugiere a ámbitos no exclusiva o no primordialmente económicos, como familia, natalidad, delincuencia y política penal"6. Foucault ubica en esta extensión del modelo general de la economía en todos los ámbitos de la gestión gubernamental al concepto que da nombre a su curso de enero de 1979: la Biopolítica. Él mismo aporta varias definiciones dispersas de lo que entiende por este término, de entre varias adelanto una que pueda servir como herramienta de discusión que aparece en el resumen del curso ya citado:

“...la manera como se ha procurado, desde el siglo XVIII, racionalizar los problemas planteados a la práctica gubernamental por los fenómenos propios de un conjunto de seres vivos constituidos como pobla-

2 Michel Foucault Seguridad, Territorio y Población, Curso del college de France (1977-1978) FCE, Buenos Aires, 2006,(1978) pp.139-160

3 Michel Foucault, “Gubernamentalidad”, Giorgio Gabriel y Fermín Rdz. Comps. Ensayos sobre biopolítica. Excesos de vida, Paidós, Buenos Aires, 2007 (1978), pp.187-215

4 Michel Foucault, El Nacimiento de la Biopolítica, Curso del College de France, FCE, Buenos Aires 2007(1979), pp.93-122

$5 \quad$ Nacimiento de la Biopolítica, p.94

$6 \quad$ Nacimiento de la Biopolítica p.365

Revista Política, Globalidad y Ciudadanía, Vol. 4 No. 7, Enero - Junio 2018, Universidad Autónoma de Nuevo León, Monterrey, México, ISSN 2395-8448. pp 31-41.. http://revpoliticas.uanl.mx/index.php/RPGyC/article/view/87 
ción: salud, higiene, natalidad, longevidad, razas..."7

El paso a las sociedades de control que funda el arte de gobernar o las tecnologías llamadas de gubernamentalidad se caracteriza por un denodado esfuerzo del control de las poblaciones en todos los ámbitos posibles, esfuerzo apuntalado por una invasión continua y sistemática de la racionalidad económica (liberal) en dichos ámbitos. En lo que sigue trataré de engranar la discusión foucaultiana de biopolítica y biopoder con debates que han derivado de esta concepción pero que se desmarcan en varios puntos de la concepción general del autor francés. Trataré de ubicar las continuidades y las rupturas en la discusión temática de varios autores que han dado un uso divergente de estos conceptos para referir a ámbitos de realidad distintos a los que refirieron en su concepción en las alocuciones del College de France.

\section{2.- FUNDAMENTO TEÓRICO}

\section{El Biopoder y la Nuda vida}

En su discusión sobre los estados de excepción, el politólogo italiano Giorgio Agamben utiliza el concepto de biopoder aplicado a una noción retomada del filósofo marxista Walter Benjamín, la de "Nuda Vida" ${ }^{8}$ Agamben utiliza la concepción general de biopoder para explicar la forma en que se establecieron estados de excepción en el mundo del siglo XX como paradigma general de gobierno, una vez más referido geográficamente a Europa Occidental. Estos estados de excepción tienen en el estado alemán de 1933-1945 y en los campos de concentración nazis, un paradigma del más alto grado de excepción como regla9 ${ }^{9}$ La nuda vida hace referencia a la vida reducida a su cualidad biológica, como vida desechable. Refiere a una figura del antiguo derecho romano que calificaba como Homo Sacer a aquella persona que no calificaba para ser sacrificada a los dioses, por lo tanto, su asesinato no obtenía la calidad de delito, era matable, desechable ${ }^{10}$.

Los Lager nazis son para este autor el modelo del estado de excepción como campo de indefinición en el que los sujetos son despojados de todo derecho y cualidad de ciudadanos (bios) y reducidos a la zoé o vida biológica. Esto los convierte en materia humana disponible, de ahí la matanza, la experimentación médica utilizando como conejillos de indias a los internos de los campos y otras formas de mortificación de la vida y de los cuerpos ${ }^{11}$.

El paradigma de los campos de concentración como campos de exterminio en donde la regla era la ausencia del derecho entendido en su forma liberal de derechos políticos y civiles individuales es extrapolado por Agamben como la forma de los estados que se construirán en el futuro con los campos de concentración como modelo trágico y destino inevitable. La relación con Foucault de Agamben se da en torno al concepto del estado de excepción como forma de gobernar la vida, pero a diferencia del primero, el exemplae histórico que propone como materia de análisis es el de un estado nacional, que se constituye en una gran fábrica de muerte, lo que lo aleja de la concepción general y de la forma de análisis foucaultiano de los micro poderes.

\footnotetext{
$7 \quad$ Ibid, p.359

8 El concepto es utilizado en Walter Benjamín, "Para la crítica de la violencia" en Angelus Novus, Edhasa, Barcelona, 1971, p.194

9 Giorgio Agamben Estado de Excepción. Homo Sacer II, Pre-textos, Valencia, 2003

10 Giorgio Agamben Homo sacer I. El poder soberano y la Nuda Vida, Pre-textos, Valencia, 1998

11 Giorgio Agamben Lo que queda de Auschwitz. El archivo y el testigo. Homo Sacer III, Pre-textos, Valencia
}

2000

Revista Política, Globalidad y Ciudadanía, Vol. 4 No. 7, Enero - Junio 2018, Universidad Autónoma de Nuevo León, Monterrey, México, ISSN 2395-8448. pp 31-41. http://revpoliticas.uanl.mx/index.php/RPGyC/article/view/87 
La biopolítica no es un efecto de la pluralidad de formas que adquiere el poder sino una forma específica en que el poder estatal se transforma en una máquina de biopoder que transforma a los sujetos en vida biológica inerme, despojada de la cualidad humana colectiva que solo le confiere su constitución en sujeto político.

La concepción de Agamben es muy sugerente por cuanto provee un ejemplo históricamente situado de las consecuencias de la biopolítica, a diferencia de lo que en Foucault es una discusión general sin referentes empíricos claros. Pero esta forma de análisis contiene limitaciones que es importante señalar y que vistos a la luz de determinantes históricos y geográficos adquieren un nuevo sentido de interpretación.

\section{Necropolítica y Colonialidad}

El biopoder y la biopolítica son una expresión contemporánea de las formas mundiales de control de la vida mediante su utilización o simbiosis con la producción de mercancías y el ensanchamiento de los mercados. A estos objetivos corresponde una forma específica de política como soberanía en la cual se implementan una serie de dispositivos que forman un repertorio de acciones tendientes al dominio.

El colonialismo es una forma privilegiada de ampliar los horizontes del capital hacia otras fronteras. El filósofo camerunés Achille Mbembé ha dado un giro importante a las tesis de la biopolítica y del estado de excepción, relacionado con esta problemática. La concepción de Agambén sobre los campos de exterminio nazis como forma histórica privilegiada de los estados de excepción y de biopoder, es históricamente incompleta puesto que existieron fenómenos anteriores a los campos alemanes, fuera de Europa, en donde el control y gestión de la vida (y el derecho de muerte) fueron ejercitados como política cotidiana sobre grandes extensiones de población y que implicaron formas de terror tanto o más (históricamente hablando) graves que las de los campos de exterminio nazis.

Para Mbembé, el colonialismo histórico y contemporáneo son formas de establecer estados de excepción en donde se construye a una parte de la humanidad, -los colonizados, como material humano disponible. Mbembé plantea que a través de mecanismos como el racismo, la separación y el aislamiento territorial, los habitantes de países colonizados fueron constituidos en nuda vida a manos de las naciones imperiales que los consideraban en tanto fuerza de trabajo disponible para la producción de la metrópoli.

A este fenómeno Mbembé lo bautiza como "Necropolítica" que consiste en:

“...la instrumentalización generalizada de la existencia humana y la destrucción material de los cuerpos y las poblaciones"12

El camerunés encuentra que la necro política tiene en la plantación y la colonia

dos de sus manifestaciones políticas explícitas como fenómeno de excepción. El trabajo esclavo en las plantaciones es una forma en la que la biopolítica se transforma para dar paso a una espiral de muerte:

"Como instrumento de trabajo, el esclavo tiene un precio. Como propiedad, el o ella tienen valor. Su trabajo es necesitado y usado. El esclavo es pues, mantenido vivo, pero en un estado injurioso, en un mundo fantasmal de horror y crueldad intensa...la vida esclava, es en muchos sentidos una suerte de muerte-en-vida" ${ }^{13}$.

12 Achille Mbembé, "Necropolitics" en Public Culture 15 (1):11-40, Duke University press, Durham (todas las traducciones en adelante G.G.R)

13 "Necropolitics", p.21

Revista Política, Globalidad y Ciudadanía, Vol. 4 No. 7, Enero - Junio 2018, Universidad Autónoma de Nuevo León, Monterrey, México, ISSN 2395-8448. pp 31-41.. http://revpoliticas.uanl.mx/index.php/RPGyC/article/view/87 
Los esclavos sufren una intensa violencia cotidiana gratuita que no tiene otro objeto que infundir terror. Es en este ataque constante a la vida en la que se encuentra la necro política de manera nítida. El esclavo, nos dice Mbembé sufre una triple pérdida en este proceso: la pérdida del hogar, del derecho sobre el cuerpo, y del status político, en los hechos es una forma de "expulsión de la humanidad". La plantación es pues una forma de poner en juego la vida en la que los seres humanos pierden su cualidad de vida política y se convierten en nuda vida disponible para ser desechada e intercambiada por otra que tenga un valor de cambio equivalente.

Por otra parte, el fenómeno de la colonización es producto del cruce de tres elementos según el autor africano: la biopolítica, el estado de excepción y el estado de sitio. Estos elementos se concatenan con la raza para producir una forma renovada de terror, según las palabras del autor:

La selección de razas, la prohibición de casamientos mixtos, la esterilización forzada, aún el exterminio de pueblos sometidos tiene su primer terreno de prueba en el mundo colonial. Aquí se puede ver la primera síntesis entre masacre y burocracia, esa encarnación de la racionalidad occidental. Hannah Arendt desarrolla la tesis de que existe un vínculo entre el nacionalsocialismo y el imperialismo tradicional...Lo que uno puede ver en la segunda guerra mundial es la extensión sobre los pueblos "civilizados" de los métodos previamente reservados a los "salvajes"14.

Para Mbembé existen dos formas políticas contemporáneas que sirven como ejemplo empírico de la forma en que se engranan los tres elementos arriba mencionados, el recién fenecido régimen de Apartheid en Sudáfrica, y el más actual de la ocupación de los territorios palestinos por el estado de Israel. La separación y el cercamiento eran elementos comunes a los dos casos, también en ambos había una orientación racial y de clase en la dominación ejercida por los poderosos. En el caso sudafricano las villas y asentamientos reservados para la población negra eran parte del diseño de gobierno de la forma de control de la población y sus movimientos, no había manera de salir de esos lugares o de entrar en otros, sin ser apercibido y vigilado. En el caso palestino, el estado de sitio se muestra en el control y el sometimiento habitual a castigos colectivos sin aparente sentido contra el pueblo palestino cometidos por el ejército y los colonos ilegales de Israel. Estas son formas contemporáneas de colonialidad en las que el necro poder muestra sus peores formas, pero el argumento de Mbembé es también extensivo al colonialismo tradicional y al más alejado históricamente de la conquista y colonización de América, en todos estos la vida humana es tratada y usada como vida desechable en base a la interiorización y deshumanización del otro, -e.g. el genocidio cometido contra los Pueblos Indígenas por los colonizadores españoles y portugueses.

En el fondo del planteamiento de Achille Mbembé se encuentra la idea de centrar desde la "diferencia colonial" 15 el argumento de la biopolítica y los estados de excepción y extender la crítica de los efectos perniciosos de la modernidad occidental a los pueblos colonizados por ella, y sobre todo a éstos. La modernidad de Occidente se impuso a la fuerza en buena parte del mundo y fue en el mundo no-occidental en donde se registraron los más largos y extendidos ejercicios de terror biopolítico que en algún momento tocaron el corazón imperial desde donde irradiaba a todo el mundo la razón. Desde esta perspectiva el análisis del filósofo africano avanza en un sentido descolonizador de lo que significa el horror para los sujetos de mundo poscolonial y saca la discusión del ámbito de intelectuales metropolitanos como Foucault y -Agamben, planteando la posibilidad de que sus argumentos tengan un verdadero carácter universal y no se los analice desde el ámbito de la diferencia imperial exclusivamente.

\footnotetext{
14 "Necropolitics" p.23

15 Walter D. Mignolo "El pensamiento des-colonial. Desprendimiento y apertura. Un manifiesto" Ponencia presentada en el Centro de Estudios Avanzados de la Universidad de Coimbra, Portugal (Primavera 2006) http://redalyc. uaemex.mx/ Tristestópicos
}

Revista Política, Globalidad y Ciudadanía, Vol. 4 No. 7, Enero - Junio 2018, Universidad Autónoma de Nuevo León, Monterrey, México, ISSN 2395-8448. pp 31-41. http://revpoliticas.uanl.mx/index.php/RPGyC/article/view/87 


\section{Las formas del Necrocapitalismo}

Esta razón, sin embargo, era producto -y en esto traigo a Foucault otra vez a la mesa- de un modo de organizar la sociedad fundado en la preeminencia del modo de producción económica y de su disciplina, la economía política en todas las esferas de la vida humana. El capitalismo es un modo de producir en la sociedad que invade esferas y mundos de la vida de manera sistemática, la producción de la muerte y la sistematización del terror y la tortura se encuentran imbricados en el modelo de racionalidad de la empresa capitalista, pero no es sino en el modelo del imperialismo clásico y del establecimiento de colonias y de formas de acumulación colonial, poscolonial o neocolonial que estos fenómenos adquieren una diáfana nitidez. Los procesos de colonización tienen una duración más allá de las luchas de liberación de los pueblos y se traducen en prácticas de sometimiento internas y externas.

S.B Banerjee acuña un término para referir a estas realidades en base al cruze del análisis de los autores citados aquí (Foucault, Agamben, Mbembé) con la escuela de economía política marxista y propone un concepto-síntesis de dos tradiciones radicales pero contrapuestas, Necrocapitalismo, al que es necesario entender como una práctica que: Opera a través del establecimiento de soberanías coloniales...y de cómo estas soberanías son establecidas en la economía política actual, donde el negocio de la muerte puede ser establecido a través de estados de excepción...la necro política se interesa en la producción de la muerte, o la subyugación de la vida al poder de la muerte, entonces es posible hablar de una necro economía -un espacio de dejar vivir o exponer a la muerte ${ }^{16}$.

El elemento del colonialismo y del papel jugado por la empresa multinacional como actor estelar del necro capitalismo planteado por Banerjee contiene dos elementos importantes a analizar, la acumulación por desposesión y los estados neoliberales de excepción.

La acumulación por desposesión es un concepto entresacado de un segmento poco atendido del tomo I de El Capital de Marx, sobre la acumulación originaria ${ }^{17}$. El geógrafo David Harvey ha desarrollado la temática sobre la acumulación capitalista y sus prolegómenos en base a esta discusión de Marx, pero reactualizándola a fenómenos contemporáneos y la ha llamado acumulación por desposesión, ésta consiste en ...encontrar nuevos campos para la acumulación de capital. Uno de los mayores impedimentos para esta acumulación era el hecho de que el capital no se podía introducir en la salud, en la educación, en la vivienda pública, así que parte del programa de flexibilización (neoliberal) era convertir todo esto en una mercancía para que el capital privado pudiese gestionarla. Después se privatizó el agua, todo tipo de actividades estatales públicas, y finalmente llegó el desmantelamiento del estado de bienestar. Pienso que deberíamos ver esto como parte de un largo proceso histórico que podemos llamar, en referencia a las privatizaciones en el campo británico, el "enclosure of the commons", el cierre de los campos comunales, la privatización de la propiedad comunal. Notad que, con el neoliberalismo, el capital utiliza a los gobiernos para privatizar, y curiosamente en el siglo XVIII también fue el gobierno británico quien cerró los "commons". Como la privatización abre nuevas oportunidades para la acumulación, los capitalistas pueden decir que tenemos una economía muy dinámica, pero el precio que hay que pagar por ello es que la gente pierde sus derechos comunales en todos los dominios que se privatizan. Por esa razón le llamo "acumulación por desposesión"18.

16 S.B Banerjee "Live and Let die. Colonial modernities and the Death worlds of Necrocapitalism" ponencia presentada en el Taller de Estudios de Gerencia Crítica, Atlanta, agosto 2006

17 Karl Marx, cap. XXIV, "La llamada acumulación originaria”, en El Capital, Tomo I, ed. FCE, México, 1972 (1946) pp.607-649

18 Araceli Varela y Marcos Bariño “Los nuevos rostros del imperialismo. Entrevista a David Harvey” en Herramienta. Revista de Debate y Crítica Marxista, www.herramienta.org

Revista Política, Globalidad y Ciudadanía, Vol. 4 No. 7, Enero - Junio 2018, Universidad Autónoma de Nuevo León, Monterrey, México, ISSN 2395-8448. pp 31-41.. http://revpoliticas.uanl.mx/index.php/RPGyC/article/view/87 
La búsqueda de nuevos terrenos para la acumulación de capital o de terrenos antes vedados a la mercantilización y a la creación de valor son parte del entramado necro capitalista en términos de la diferencia colonial, las metrópolis imperiales (nuevas y antiguas) requieren de nuevos campos para la acumulación y sus colonias o satélites los pueden proveer través de la Privatización de la tierra y el subsecuente desplazamiento forzado de campesinos, la conversión de propiedad pública en privada, las restricciones al uso público de recursos de propiedad común, las practicas neocoloniales de apropiación de bienes, el control de los recursos naturales en las ex-colonias, y la supresión de formas indígenas alternativas de consumo y producción $^{19}$.

Para Banerjee la acumulación por desposesión es un elemento inseparable de los nuevos diseños del capital en el mundo de la economía global. Este fenómeno de acumulación que deprecia la vida en forma sistemática requiere un tipo de soberanía y de tecnologías de gobierno adecuados para sus fines. Banerjee recurre al trabajo teórico y etnográfico de la antropóloga feminista malaya Aihwa Ong para explicar el diseño específico de gobierno correspondiente a las necesidades del capital ya mencionadas, haciendo acopio de las categorías acuñadas por ella, como "soberanía gradual" y "neoliberalismo como excepción"20 explica como en el neoliberalismo asiático se ha diseñado un trato diferencial para poblaciones fragmentadas de por sí (por raza, clase, etnia, género y región) como producto de la relación de los estados con el capital trasnacional, este trato diferenciado se acompaña asimismo por una reconfiguración del poder a manos de corporaciones trasnacionales en zonas especiales de procesamiento de exportaciones (como en las zonas de maquiladoras en la frontera norte de México). Como resultado de esta interacción se crean áreas diferenciadas de soberanía; en algunas hay una presencia fuerte del estado y en otras mandas el mercado global y el capital extranjero "la soberanía estatal se dispersa porque el capital y los mercados globales con la colusión de los gobiernos crean estados de excepción en donde priva la coerción, la violencia y el asesinato"21.

Asimismo, los territorios son "limpiados" de rebeldes (ciudadanos fuera de la ley) para dar paso a concesiones, oleoductos y minas. El papel del estado en este sentido se reduce a resguardar ciertos derechos democráticos en la esfera estrictamente política, mientras en la esfera económica estos mismos derechos son socavados de manera sistemática por la explotación y la violencia.

Poblaciones dispensables en el mundo de la neocolonialidad

Desde otros registros discursivos se ha dado cuenta a lo largo de la historia de los procesos arriba descritos, pero en base a interpretaciones lejanas a la tradición posestructuralista. Desde el marxismo la problemática de los países descolonizados de África ha sido analizado desde la lógica de acumulación de capital y las transformaciones estructurales del patrón mundial de acumulación de capital. El antropólogo francés Claude Meillassoux llega a conclusiones en mucho análogas a las planteadas por Banerjee dando cuenta de las formas de utilización instrumental de la vida como parte de los procesos de reacomodo mundial del capital, en lo que refiere a la demografía de la población africana y a los gritos de alarma lanzados desde las metrópolis y los organismos multilaterales acerca de la superpoblación de ese continente.

Meillassoux describe el proceso por el cual los países africanos merced a procesos históricos de desarrollo capitalista de los cuales tienen poco o ningún control, son colocados en una situación de dependencia alimentaria, al mismo tiempo que crece su población. De manera paralela el trabajo en sus países se pierde cada vez más, y la demanda de mano de obra en los países centrales disminuye, Meillassoux plantea que

\footnotetext{
19 S.B Banerjee, "Live and Let die..." Op. cit. p.9

20 Brett Neilson y Gigi Roggero "Neoliberismo comme una tecnologia di governo, economia sovranità e cittadinanza. Un'intervista con Aihwa Ong” Il manifesto Quotidiano Comunista http://www.ilmanifesto.it traducción GGR 21 "Live and Let die"... p.12

Revista Política, Globalidad y Ciudadanía, Vol. 4 No. 7, Enero - Junio 2018, Universidad Autónoma de Nuevo León, Monterrey,
} México, ISSN 2395-8448. pp 31-41. http://revpoliticas.uanl.mx/index.php/RPGyC/article/view/87 
...la población que creció en estos decenios (1950-1970) bajo los efectos de una política de importación alimenticia y de existencia de empleos está hoy en una situación de sobrepoblación relativa. El problema para la economía capitalista que la creó, es hacerla desaparecer sin hacerse cargo. Ideológicamente se la presenta como una superpoblación absoluta, confundiendo la población actual con las predicciones de una futura población gigantesca y aterrorizante, pero en este momento imaginaria...el control de la demografía de los pueblos explotados por medios demográficos (control de la natalidad, esterilización, etc.) fracasó. Por tanto, se establece una forma de control mediante el hambre, la enfermedad y la muerte, más eficaz y más cruel, con el pretexto de la "racionalidad económica" y el "ajuste estructural": la lección de Malthus fue comprendida ${ }^{22}$.

El diagnóstico sobre el futuro que espera a las poblaciones de África, de terror y muerte, parece validarse de manera constante cuando escuchamos de una nueva masacre, de abusos cometidos contra sectores vulnerables (mujeres y niñ@s), de una nueva plaga de hambre en otro país de África. La política de muerte que el capitalismo tiene reservada a sectores de la población mundial que se consideran irrelevantes es una forma de vida desnuda anterior al nazismo y que lo sobrevive hasta esta fecha. Para el África y para otras poblaciones del mundo periférico esta ha sido una constante de vida que solo ha tenido respiros históricos intermitentes.

\section{Un caso de excepcionalidad latinoamericana}

Un caso de surgimiento de amplios sectores de población como vida desnuda y del establecimiento de la excepcionalidad como norma de vida es el caso de Colombia, desde hace decenios, pero agravado con lo que con el último gobierno se ha llamado "política de seguridad democrática". Diversas organizaciones de víctimas de la violencia estatal han denunciado el carácter esencialmente represivo de este diseño político de gobierno y la relación de imbricación entre los más altos mandos del gobierno, la policía y e 1 ejército con los carteles del narcotráfico y especialmente con los grupos paramilitares de derecha.

De manera tal que la seguridad democrática tiene un único objetivo, acabar con las organizaciones insurgentes armadas, y en primer lugar con las FARC y suprimir por el medio que sea a las organizaciones sociales, sindicales y populares que luchan contra las políticas del gobierno, haciendo tabla rasa de todas ellas, identificándolas como "colaboradores de la guerrilla" lo que equivale a declararlos vida inservible, matable. Ningún estado latinoamericano tiene el registro que tiene Colombia de desapariciones forzadas, y ejecuciones extrajudiciales, además del desplazamiento forzado de sectores enteros de población campesina e indígena por la acción genocida de las Autodefensas Unidas de Colombia (AUC), el principal grupo paramilitar.

Se ha registrado incluso la forma en que las AUC son contratadas por empresas multinacionales, ganaderos y terratenientes y narcotraficantes para hacerse de tierras en determinados sectores geográficos, ya no como producto de un conflicto entre distintas organizaciones civiles armadas, sino como parte de una planeación deliberada de acumulación por desposesión ${ }^{23}$. Para las empresas, los paramilitares aseguran un "entorno seguro para los negocios" mediante la desaparición y asesinato de dirigentes sindicales y de obreros, y en el caso de desplazamientos forzados, la tierra que ha sido abandonada es concedida a terratenientes para el cultivo de productos agroindustriales o la ganadería. De esa forma avanzan las "inversiones" y los

22 Claude Meillassoux L'economie de la vie. Demographie du travail ed. Page Deux, Paris, 1997, pp.106-109, traducción GGR

23 Cfr. Iván Cepeda-Castro "Los paramilitares: Dispositivo del modelo "democrático" de control social" en sitio digital del equipo Nizkor, 29 de septiembre 2003, Internet, ver http://www.derechos.org/nizkor/colombia/doc/cepeda8. html

Revista Política, Globalidad y Ciudadanía, Vol. 4 No. 7, Enero - Junio 2018, Universidad Autónoma de Nuevo León, Monterrey, México, ISSN 2395-8448. pp 31-41.. http://revpoliticas.uanl.mx/index.php/RPGyC/article/view/87 
negocios en ese país, mediante la siembre del terror para la población que se insubordina.

Paradójicamente todo esto ocurre en un entorno de democracia y elecciones libres y de existencia formal del estado de derecho. De la manera como ONG ha estudiado para Asia la forma en que en el neoliberalismo existen formas graduales de soberanía y en algunos sectores el estado simplemente ha acordado no hacer nada para hacer cumplir la ley o ha impuesto una forma de legalidad de excepción que permite mediante la ambigüedad una manga ancha para permitir vivir o dar la muerte. Así en Colombia, el estado tolera y solapa a los cárteles del narcotráfico y a las AUC, porque son parte de su diseño de gobierno de la población y herramienta de las clases dominantes nativas y del capital transnacional para abrir nuevas zonas a la acumulación y ensanchar el horizonte de sus ganancias.

\section{3.- MÉTODO}

Diseño

La discusión contenida en esta investigación documental se enmarca en la tradición cualitativa interpretativa de los debates teóricos en Ciencias Sociales (Álvarez-Gayou, 2003; Vasilachis, 2006) referidas al estado, a su caracterización y sus transformaciones (Osorio, 2004; Abrams, Gupta y Mitchell, 2015). Se encuadra en las concepciones de investigación naturalista no-experimental de los fenómenos sociopolíticos con un alcance de nivel exploratorio (Borsotti, 2007) de la construcción de nuevas interrogantes sobre la naturaleza del estado en el contexto de las grandes transformaciones ocurridas desde el último tercio del siglo XX.

Instrumentos

Para la construcción del marco teórico-conceptual del tema la transformación biopolitica del Estado-Nación, se consultaron un total de veinte referencias bibliográficas utilizándose como instrumento las ideas, argumentos y proyectos que fueron interpretados desde una perspectiva analítica y crítica.

\section{Procedimiento}

Con la intención de realizar una comprensión del problema de la investigación se recopilaron fuentes secundarias de documentos académicos. En el marco de referencia se definen los conceptos básicos con relación a Biopolítica y Estado Nación. Una vez recopilada y analizada la información se construyó el documento objeto de este trabajo. Finalmente se construyeron una serie de conclusiones relativas a los objetivos trazados

\section{4.- CONCLUSIONES}

De manera harto coincidente el marxismo y la tradición que proviene del posestructuralismo plantean diagnósticos muy emparentados acerca de la forma en que los diseños del poder crean tecnologías de gobierno o esferas de dominación para controlar la vida humana sea para hacerla florecer o para extinguirla, según el momento histórico y el grupo específico de población que se trate. Existen, sin embargo, diferencias que es imposible soslayar, respecto del lugar de enunciación (la diferencia imperial o la diferencia colonial), respecto del compromiso político con los sujetos de las investigaciones y respecto de la conveniencia o no de plantear caminos emancipatorios o prescripciones para luchar contra este tipo de diseño de poder. Desde la visión foucaultiana, no es deseable plantear otro diseño de poder, pues este podría desembocar igualmente en otra forma opresiva. No así desde la visión general del marxismo, que plantea a la investigación como una parte de la tarea política de lucha contra la explotación y de liberación de los oprimidos.

La adscripción de cada investigador o académico es más bien parte de una decisión ética y un compromiso político como sujetos. Por lo pronto, algunos de los autores revisados aquí dan cuenta del significado de las Ciencias Sociales como crítica de lo existente y proveen herramientas para aquellos que desde otros espacios plantean la lucha por un mundo en donde la muerte no sea el destino inexorable para muchos seres

Revista Política, Globalidad y Ciudadanía, Vol. 4 No. 7, Enero - Junio 2018, Universidad Autónoma de Nuevo León, Monterrey, México, ISSN 2395-8448. pp 31-41. http://revpoliticas.uanl.mx/index.php/RPGyC/article/view/87 
humanos.

\section{REFERENCIAS}

Abrams, P., Gupta. A y Mitchell, T. (2015) Antropología del Estado, México, Fondo de Cultura Económica Agamben, G. (1998) Homo sacer I. El poder soberano y la Nuda Vida, Valencia, Pre-Textos

Agamben, G. (2000) Lo que queda de Auschwitz. El archivo y el testigo. Homo Sacer III, Valencia, PreTextos

Agamben, G. (2003) Estado de Excepción. Homo Sacer II, Valencia, Pre-Textos

Alvarez-Gayou, J.L (2003) Cómo hacer investigación cualitativa. Fundamentos y Metodologia Mexico, Paidós

Banerjee, S. (2006) "Live and Let die. Colonial modernities and the Death worlds of Necrocapitalism" ponencia presentada en el Taller de Estudios de Gerencia Crítica, Atlanta, Agosto.

Benjamin, W. (1971) "Para la crítica de la violencia” en Angelus Novus, Barcelona, Edhasa p.194

Borsotti, C.A (2007) Temas de metodología de la investigación en Ciencias Sociales empíricas, Buenos Aires, Miño y Dávila

Cepeda-Castro, I. (2003). Los paramilitares: Dispositivo del modelo "democrático" de control social. en sitio digital del equipo Nizkor, 29 de septiembre, Recuperado de http://www.derechos.org/nizkor/ colombia/doc/cepeda8.html

Foucault, M. (2006 (1978)) Seguridad, Territorio y Población, Curso del College de France (1977-1978) FCE, Buenos Aires, pp.139-160

Foucault, M. (2007a) “Gubernamentalidad”, en Giorgio Gabriel y Fermín Rodríguez Comps. Ensayos sobre biopolítica. Excesos de vida, Buenos Aires, Paidós pp.187-215

Foucault, M. (2007b) El Nacimiento de la Biopolítica, Curso en el College de France 1978-1979, Buenos Aires, FCE, pp.93-122

Bascón, M.: Cazallo, A.; Lechuga, J. \& Meñaca, I. (2016). Estudio de la necesidad de implantar un servicio público de transporte entre las ciudades de Ceuta-Tetuán y Melilla-Nador. En Desarrollo Gerencial Revista de la Facultad de Ciencias Económicas, administrativas y contables de la Universidad Simón Bolívar 8(2), 37 - 57.

Benjamin, W. (1971) "Para la crítica de la violencia" en Angelus Novus, Edhasa, Barcelona, p.194

Mbembé, A.(2003) "Necropolitics" en Public Culture 15 (1):11-40, Duke University press, Durham .

Marx, K. (1972 (1946)) El Capital, Tomo I, ed. FCE, cap. XXIV, "La llamada acumulación originaria”, Mexico, pp.607-649

Meillassoux, C. (1997) L'economie de la vie. Demographie du travail ed. Page Deux, Paris, pp.106-109

Mignolo, W. (2006). El pensamiento des-colonial. Desprendimiento y apertura. Un manifiesto" Ponencia presentada en el Centro de Estudios Avanzados de la Universidad de Coimbra, Portugal (Primavera). Recuperado de http://redalyc.uaemex.mx/ Tristestópicos

Neilson, B. y Gigi Roggero (2007). Neoliberismo comme una tecnologia di governo, economia sovranità e cittadinanza. Un'intervista con Aihwa Ong. Il manifesto Quotidiano Comunista, 14 de marzo. Recuperado de http://www.ilmanifesto.it

Revista Política, Globalidad y Ciudadanía, Vol. 4 No. 7, Enero - Junio 2018, Universidad Autónoma de Nuevo León, Monterrey, México, ISSN 2395-8448. pp 31-41.. http://revpoliticas.uanl.mx/index.php/RPGyC/article/view/87 
Varela, A. y Bariño, M. (año). Los nuevos rostros del imperialismo. Entrevista a David Harvey en Herramienta. Revista de Debate y Crítica Marxista . Recuperado de www.herramienta.org

Vasilachis de Gialdino, I. (coord.) (2006) Estrategias de Investigacion Cualitativa, Barcelona, Gedisa, 2006 